\title{
PROJECTION ITERATIVE APPROXIMATIONS FOR A NEW CLASS OF GENERAL RANDOM IMPLICIT QUASI-VARIATIONAL INEQUALITIES
}

\author{
HENG-YOU LAN
}

Received 9 November 2005; Accepted 21 January 2006

We introduce a class of projection-contraction methods for solving a class of general random implicit quasi-variational inequalities with random multivalued mappings in Hilbert spaces, construct some random iterative algorithms, and give some existence theorems of random solutions for this class of general random implicit quasi-variational inequalities. We also discuss the convergence and stability of a new perturbed Ishikawa iterative algorithm for solving a class of generalized random nonlinear implicit quasivariational inequalities involving random single-valued mappings. The results presented in this paper improve and extend the earlier and recent results.

Copyright (c) 2006 Heng-You Lan. This is an open access article distributed under the Creative Commons Attribution License, which permits unrestricted use, distribution, and reproduction in any medium, provided the original work is properly cited.

\section{Introduction}

Throughout this paper, we suppose that $\mathbb{R}$ denotes the set of real numbers and $(\Omega, \mathscr{A}, \mu)$ is a complete $\sigma$-finite measure space. Let $E$ be a separable real Hilbert space endowed with the norm $\|\cdot\|$ and inner product $\langle\cdot, \cdot\rangle$, let $D$ be a nonempty subset of $E$, and let $C B(E)$ denote the family of all nonempty bounded closed subsets of $E$. We denote by $\mathscr{B}(E)$ the class of Borel $\sigma$-fields in $E$.

In this paper, we will consider the following new general random implicit quasivariational inequality with random multivalued mappings by using a new class of projection method: find $x: \Omega \rightarrow D$ and $u, v, \xi: \Omega \rightarrow E$ such that $g(t, x(t)) \in J(t, v(t)), u(t) \in$ $T(t, x(t)), v(t) \in F(t, x(t)), \xi(t) \in S(t, x(t))$, and

$$
a(t, u(t), g(t, y)-g(t, x(t)))+\langle N(t, f(t, x(t)), \xi(t)), g(t, y)-g(t, x(t))\rangle \geq 0
$$

for all $t \in \Omega$ and $g(t, y) \in J(t, v(t))$, where $g: \Omega \times D \rightarrow E, f: \Omega \times E \rightarrow E$, and $N: \Omega \times E \times$ $E \rightarrow E$ are measurable single-valued mappings, $T, S: \Omega \times D \rightarrow C B(E)$ and $F: \Omega \times D \rightarrow$ $C B(D)$ are random multivalued mappings, $J: \Omega \times D \rightarrow P(E)$ is a random multivalued 
mapping such that for each $t \in \Omega$ and $x \in D, P(E)$ denotes the power set of $E, J(t, x)$ is closed convex, and $a: \Omega \times E \times E \rightarrow \mathbb{R}$ is a random function.

Some special cases of the problem (1.1) are presented as follows.

If $g \equiv I$, the identity mapping, then the problem (1.1) is equivalent to the problem of finding $x: \Omega \rightarrow D$ and $u, v, \xi: \Omega \rightarrow E$ such that $x(t) \in J(t, v(t)), v(t) \in F(t, x(t)), u(t) \in$ $T(t, x(t)), \xi(t) \in S(t, x(t))$, and

$$
a(t, u(t), y-x(t))+\langle N(t, f(t, x(t)), \xi(t)), y-x(t)\rangle \geq 0
$$

for all $t \in \Omega$ and $y \in J(t, v(t))$. The problem (1.2) is called a generalized random strongly nonlinear multivalued implicit quasi-variational inequality problem and appears to be a new one.

If $J(t, x(t))=m(t, x(t))+K$, where $m: \Omega \times D \rightarrow E$ and $K$ is a nonempty closed convex subset of $E$, then the problem (1.1) becomes to the following generalized nonlinear random implicit quasi-variational inequality for random multivalued mappings: find $x: \Omega \rightarrow D$ and $u, v, \xi: \Omega \rightarrow E$ such that $g(t, x(t))-m(t, v(t)) \in K, u(t) \in T(t, x(t)), v(t) \in$ $F(t, x(t)), \xi(t) \in S(t, x(t))$, and

$$
a(t, u(t), g(t, y)-g(t, x(t)))+\langle N(t, f(t, x(t)), \xi(t)), g(t, y)-g(t, x(t))\rangle \geq 0
$$

for all $t \in \Omega$ and $g(t, y) \in m(t, v(t))+K$.

If $N(t, z, w)=w+z$ for all $t \in \Omega, z, w \in E$, then the problem (1.1) reduces to finding $x: \Omega \rightarrow D$ and $u, v, \xi: \Omega \rightarrow E$ such that $g(t, x(t)) \in J(t, v(t)), u(t) \in T(t, x(t)), v(t) \in$ $F(t, x(t)), \xi(t) \in S(t, x(t))$, and

$$
a(t, u(t), g(t, y)-g(t, x(t)))+\langle f(t, x(t))+\xi(t), g(t, y)-g(t, x(t))\rangle \geq 0
$$

for all $t \in \Omega$ and $g(t, y) \in J(t, v(t))$.

The problem (1.4) is called a generalized random implicit quasi-variational inequality for random multivalued mappings, which is studied by Cho et al. [8] when $T \equiv I$ and $f \equiv 0$, and includes various known random variational inequalities. For details, we refer the reader to $[8,11,12,19]$ and the references therein.

If $T, S: \Omega \times D \rightarrow E$ and $F: \Omega \times D \rightarrow D$ are random single-valued mappings, and $F=I$, then the problem (1.4) becomes to the following random generalized nonlinear implicit quasi-variational inequality problem involving random single-valued mappings: find $x$ : $\Omega \rightarrow D$ such that $g(t, x(t)) \in J(t, x(t))$ and

$$
a(t, T(t, x(t)), g(t, y)-g(t, x(t)))+\langle f(t, x(t))+S(t, x(t)), g(t, y)-g(t, x(t))\rangle \geq 0
$$

for all $t \in \Omega$ and $g(t, y) \in J(t, x(t))$.

Remark 1.1. Obviously, the problem (1.1) includes a number of classes of variational inequalities, complementarity problems, and quasi-variational inequalities as special cases (see, e.g., $[1,4,5,8,10-13,15,17,19,20,25]$ and the references therein). 
The study of such types of problems is inspired and motivated by an increasing interest in the nonlinear random equations involving the random operators in view of their need in dealing with probabilistic models, which arise in biological, physical, and system sciences and other applied sciences, and can be solved with the use of variational inequalities (see [21]). Some related works, we refer to $[2,4]$ and the references therein. Further, the recent research works of these fascinating areas have been accelerating the random variational and random quasi-variational inequality problems to be introduced and studied by Chang [4], Chang and Zhu [7], Cho et al. [8], Ganguly and Wadhwa [11], Huang [14], Huang and Cho [15], Huang et al. [16], Noor and Elsanousi [19], and Yuan et al. [24].

On the other hand, in [22], Verma studied an extension of the projection-contraction method, which generalizes the existing projection-contraction methods, and applied the extended projection-contraction method to the solvability of a general monotone variational inequalities. Very recently, Lan et al. $[17,18]$ introduced and studied some new iterative algorithms for solving a class of nonlinear variational inequalities with multivalued mappings in Hilbert spaces, and gave some convergence analysis of iterative sequences generated by the algorithms.

In this paper, we introduce a class of projection-contraction methods for solving a new class of general random implicit quasi-variational inequalities with random multivalued mappings in Hilbert spaces, construct some random iterative algorithms, and give some existence theorems of random solutions for this class of general random implicit quasivariational inequalities. We also discuss the convergence and stability of a new perturbed Ishikawa iterative algorithm for solving a class of generalized random nonlinear implicit quasi-variational inequalities involving random single-valued mappings. Our results improve and generalize many known corresponding results in the literature.

\section{Preliminaries}

In the sequel, we first give the following concepts and lemmas which are essential for this paper.

Definition 2.1. A mapping $x: \Omega \rightarrow E$ is said to be measurable if for any $B \in \mathscr{B}(E),\{t \in$ $\Omega: x(t) \in B\} \in \mathscr{A}$.

Definition 2.2. A mapping $F: \Omega \times E \rightarrow E$ is said to be

(i) a random operator if for any $x \in E, F(t, x)=y(t)$ is measurable;

(ii) Lipschitz continuous (resp., monotone, linear, bounded) if for any $t \in \Omega$, the mapping $F(t, \cdot): E \rightarrow E$ is Lipschitz continuous (resp., monotone, linear, bounded).

Definition 2.3. A multivalued mapping $\Gamma: \Omega \rightarrow P(E)$ is said to be measurable if for any $B \in \mathscr{B}(E), \Gamma^{-1}(B)=\{t \in \Omega: \Gamma(t) \cap B \neq \varnothing\} \in \mathscr{A}$.

Definition 2.4. A mapping $u: \Omega \rightarrow E$ is called a measurable selection of a multivalued measurable mapping $\Gamma: \Omega \rightarrow P(E)$ if $u$ is measurable and for any $t \in \Omega, u(t) \in \Gamma(t)$.

Definition 2.5. Let $D$ be a nonempty subset of a separable real Hilbert space $E$, let $g$ : $\Omega \times D \rightarrow E, f: \Omega \times E \rightarrow E$, and $N: \Omega \times E \times E \rightarrow E$ be three random mappings and let 
$T: \Omega \times D \rightarrow C B(E)$ be a multivalued measurable mapping. Then

(i) $f$ is said to be $c(t)$-strongly monotone on $\Omega \times D$ with respect to the second argument of $N$ and $g$, if for all $t \in \Omega$ and $x, y \in D$, there exists a measurable function $c: \Omega \rightarrow(0,+\infty)$ such that

$$
\langle g(t, x)-g(t, y), N(t, f(t, x), \cdot)-N(t, f(t, y), \cdot)\rangle \geq c(t)\|g(t, x)-g(t, y)\|^{2}
$$

(ii) $f$ is said to be $v(t)$-Lipschitz continuous on $\Omega \times D$ with respect to the second argument of $N$ and $g$, if for any $t \in \Omega$ and $x, y \in D$, there exists a measurable function $v: \Omega \rightarrow(0,+\infty)$ such that

$$
\|N(t, f(t, x), \cdot)-N(t, f(t, y), \cdot)\| \leq v(t)\|g(t, x)-g(t, y)\|
$$

and if $N(t, f(t, x), y)=f(t, x)$ for all $t \in \Omega$ and $x, y \in D$, then $f$ is said to be Lipschitz continuous on $\Omega \times D$ with respect to $g$ with measurable function $\nu(t)$;

(iii) $N$ is said to be $\sigma(t)$-Lipschitz continuous on $E$ with respect to the third argument if there exists a measurable function $\sigma: \Omega \rightarrow(0,+\infty)$ such that

$$
\|N(t, \cdot, x)-N(t, \cdot, y)\| \leq \sigma(t)\|x-y\|, \quad \forall x, y \in E
$$

(iv) $T$ is said to be $H$-Lipschitz continuous on $\Omega \times D$ with respect to $g$ with measurable function $\gamma(t)$ if there exists a measurable function $\gamma: \Omega \rightarrow(0,+\infty)$ such that for any $t \in \Omega$ and $x, y \in D$,

$$
H(T(t, x), T(t, y)) \leq \gamma(t)\|g(t, x)-g(t, y)\|
$$

where $H(\cdot, \cdot)$ is the Hausdorff metric on $C B(E)$ defined as follows: for any given $A, B \in C B(E)$,

$$
H(A, B)=\max \left\{\sup _{x \in A} \inf d(x, y), \sup _{y \in B} \inf d(x, y)\right\} .
$$

Definition 2.6. A random multivalued mapping $S: \Omega \times E \rightarrow P(E)$ is said to be

(i) measurable if, for any $x \in E, S(\cdot, x)$ is measurable;

(ii) $H$-continuous if, for any $t \in \Omega, S(t, \cdot)$ is continuous in the Hausdorff metric.

Lemma 2.7 [3]. Let $M: \Omega \times E \rightarrow C B(E)$ be an $H$-continuous random multivalued mapping. Then for any measurable mapping $x: \Omega \rightarrow E$, the multivalued mapping $M(\cdot, x): \Omega \rightarrow$ $C B(E)$ is measurable.

Lemma 2.8 [3]. Let $M, V: \Omega \times E \rightarrow C B(E)$ be two measurable multivalued mappings, let $\epsilon>0$ be a constant, and let $x: \Omega \rightarrow E$ be a measurable selection of $M$. Then there exists $a$ 
measurable selection $y: \Omega \rightarrow$ E of $V$ such that

$$
\|x(t)-y(t)\| \leq(1+\epsilon) H(M(t), V(t)), \quad \forall t \in \Omega .
$$

Definition 2.9. An operator $a: \Omega \times E \times E \rightarrow E$ is called a random $\beta(t)$-bounded bilinear function if the following conditions are satisfied:

(1) for any $t \in \Omega, a(t, \cdot, \cdot)$ is bilinear and there exists a measurable function $\beta: \Omega \rightarrow$ $(0,+\infty)$ such that

$$
|a(t, x, y)| \leq \beta(t)\|x\| \cdot\|y\|, \quad \forall t \in \Omega, x, y \in E
$$

(2) for any $x, y \in E, a(\cdot, x, y)$ is a measurable function.

LEMMA 2.10 [15]. If $a$ is a random bilinear function, then there exists a unique random bounded linear operator $A: \Omega \times E \rightarrow$ E such that

$$
\langle A(t, x), y\rangle=a(t, x, y), \quad\|A(t, \cdot)\|=\|a(t, \cdot, \cdot)\|
$$

for all $t \in \Omega$ and $x, y \in E$, where

$$
\begin{gathered}
\|A(t, \cdot)\|=\sup \{\|A(t, x)\|:\|x\| \leq 1\}, \\
\|a(t, \cdot, \cdot)\|=\sup \{|a(t, x, y)|:\|x\| \leq 1,\|y\| \leq 1\} .
\end{gathered}
$$

Lemma 2.11 [4]. Let $K$ be a closed convex subset of $E$. Then for an element $z \in K, x \in K$ satisfies the inequality $\langle x-z, y-x\rangle \geq 0$ for all $y \in K$ if and only if

$$
x=P_{K}(z)
$$

where $P_{K}$ is the projection of $E$ on $K$.

It is well known that the mapping $P_{K}$ defined by (2.10) is nonexpansive, that is,

$$
\left\|P_{K}(x)-P_{K}(y)\right\| \leq\|x-y\|, \quad \forall x, y \in E .
$$

Definition 2.12. Let $J: \Omega \times D \rightarrow P(E)$ be a random multivalued mapping such that for each $t \in \Omega$ and $x \in E, J(t, x)$ is a nonempty closed convex subset of $E$. The projection $P_{J(t, x)}$ is said to be a $\tau(t)$-Lipschitz continuous random operator on $\Omega \times D$ with respect to $g$ if

(1) for any given $x, z \in E, P_{J(t, x)}(z)$ is measurable;

(2) there exists a measurable function $\tau: \Omega \rightarrow(0,+\infty)$ such that for all $x, y, z \in E$ and $t \in \Omega$,

$$
\left\|P_{J(t, x)}(z)-P_{J(t, y)}(z)\right\| \leq \tau(t)\|g(t, x)-g(t, y)\| .
$$

If $g(t, x)=x$ for all $x \in D$, then $P_{J(t, x)}$ is said to be a $\tau(t)$-Lipschitz continuous random operator on $\Omega \times D$. 
Lemma 2.13 [5]. Let $K$ be a closed convex subset of $E$ and let $m: \Omega \times E \rightarrow E$ be a random operator. If $J(t, x)=m(t, x)+K$ for all $t \in \Omega$ and $x \in E$, then

(i) for any $z \in E, P_{J(t, x)}(z)=m(t, x)+P_{K}(z-m(t, x))$ for all $t \in \Omega$ and $x \in E$;

(ii) $P_{J(t, x)}$ is a $2 \kappa(t)$-Lipschitz continuous operator when $m$ is a $\kappa(t)$-Lipschitz continuous random operator.

\section{Existence and convergence theorems}

In this section, we suggest and analyze a new projection-contraction iterative method for solving the random multivalued variational inequality (1.1). Firstly, from the proof of Theorem 3.2 in Cho et al. [9], we have the following lemma.

Lemma 3.1. Let $D$ be a nonempty subset of a separable real Hilbert space $E$ and let $J$ : $\Omega \times D \rightarrow P(E)$ be a random multivalued mapping such that for each $t \in \Omega$ and $x \in E, J(t, x)$ is a closed convex subset in $E$ and $J(\Omega \times D) \subset g(\Omega \times D)$. Then the measurable mappings $x: \Omega \rightarrow D$ and $u, v, \xi: \Omega \rightarrow E$ are the solutions of (1.1) if and only if for any $t \in \Omega, u(t) \in$ $T(t, x(t)), v(t) \in F(t, x(t)), \xi(t) \in S(t, x(t))$, and

$$
g(t, x(t))=P_{J(t, v(t))}[g(t, x(t))-\rho(t)(N(t, f(t, x(t)), \xi(t))+A(t, u(t)))],
$$

where $\rho: \Omega \rightarrow(0,+\infty)$ is a measurable function and $\langle A(t, x), y\rangle=a(t, x, y)$ for all $t \in \Omega$ and $x, y \in E$.

Based on Lemma 3.1, we are now in a position to propose the following generalized and unified new projection-contraction iterative algorithm for solving the problem (1.1).

Algorithm 3.2. Let $D$ be a nonempty subset of a separable real Hilbert space $E$ and let $\lambda: \Omega \rightarrow(0,1)$ be a measurable step size function. Let $g: \Omega \times D \rightarrow E, f: \Omega \times E \rightarrow E$, and $N: \Omega \times E \times E \rightarrow E$ be measurable single-valued mappings. Let $T, S: \Omega \times D \rightarrow C B(E)$ and $F: \Omega \times D \rightarrow C B(D)$ be multivalued random mappings. Let $J: \Omega \times D \rightarrow P(E)$ be a random multivalued mapping such that for each $t \in \Omega$ and $x \in D, J(t, x)$ is closed convex and $J(\Omega \times D) \subset g(\Omega \times D)$. Then by Lemma 2.7 and Himmelberg [12], we know that for given $x_{0}(\cdot) \in D$, the multivalued mappings $T\left(\cdot, x_{0}(\cdot)\right), F\left(\cdot, x_{0}(\cdot)\right)$, and $S\left(\cdot, x_{0}(\cdot)\right)$ are measurable, and there exist measurable selections $u_{0}(\cdot) \in T\left(\cdot, x_{0}(\cdot)\right), v_{0}(\cdot) \in F\left(\cdot, x_{0}(\cdot)\right)$, and $\xi_{0}(\cdot) \in S\left(\cdot, x_{0}(\cdot)\right)$. Set $g\left(t, x_{1}(t)\right)=g\left(t, x_{0}(t)\right)-\lambda(t)\left\{g\left(t, x_{0}(t)\right)-P_{J\left(t, v_{0}(t)\right)}\left[g\left(t, x_{0}(t)\right)-\right.\right.$ $\left.\left.\rho(t)\left(N\left(t, f\left(t, x_{0}(t)\right), \xi_{0}(t)\right)+A\left(t, u_{0}(t)\right)\right)\right]\right\}$, where $\rho$ and $A$ are the same as in Lemma 3.1. Then it is easy to know that $x_{1}: \Omega \rightarrow E$ is measurable. Since $u_{0}(t) \in T\left(t, x_{0}(t)\right) \in C B(E)$, $v_{0}(t) \in F\left(t, x_{0}(t)\right) \in C B(D)$, and $\xi_{0}(t) \in S\left(t, x_{0}(t)\right) \in C B(E)$, by Lemma 2.8, there exist measurable selections $u_{1}(t) \in T\left(t, x_{1}(t)\right), v_{1}(t) \in F\left(t, x_{1}(t)\right)$, and $\xi_{1}(t) \in S\left(t, x_{1}(t)\right)$ such that for all $t \in \Omega$,

$$
\begin{gathered}
\left\|u_{0}(t)-u_{1}(t)\right\| \leq\left(1+\frac{1}{1}\right) H\left(T\left(t, x_{0}(t)\right), T\left(t, x_{1}(t)\right)\right), \\
\left\|v_{0}(t)-v_{1}(t)\right\| \leq\left(1+\frac{1}{1}\right) H\left(F\left(t, x_{0}(t)\right), F\left(t, x_{1}(t)\right)\right), \\
\left\|\xi_{0}(t)-\xi_{1}(t)\right\| \leq\left(1+\frac{1}{1}\right) H\left(S\left(t, x_{0}(t)\right), S\left(t, x_{1}(t)\right)\right) .
\end{gathered}
$$


By induction, we can define sequences $\left\{x_{n}(t)\right\},\left\{u_{n}(t)\right\},\left\{v_{n}(t)\right\}$, and $\left\{\xi_{n}(t)\right\}$ inductively satisfying

$$
\begin{aligned}
g\left(t, x_{n+1}(t)\right)=g\left(t, x_{n}(t)\right)-\lambda(t)\left\{g\left(t, x_{n}(t)\right)-P_{J\left(t, v_{n}(t)\right)}\left[g\left(t, x_{n}(t)\right)\right.\right. & \left.\left.-\rho(t)\left(N\left(t, f\left(t, x_{n}(t)\right), \xi_{n}(t)\right)+A\left(t, u_{n}(t)\right)\right)\right]\right\}, \\
& u_{n}(t) \in T\left(t, x_{n}(t)\right), \\
\left\|u_{n}(t)-u_{n+1}(t)\right\| \leq\left(1+\frac{1}{n+1}\right) H\left(T\left(t, x_{n}(t)\right), T\left(t, x_{n+1}(t)\right)\right), & v_{n}(t) \in F\left(t, x_{n}(t)\right), \\
\left\|v_{n}(t)-v_{n+1}(t)\right\| \leq & \left(1+\frac{1}{n+1}\right) H\left(F\left(t, x_{n}(t)\right), F\left(t, x_{n+1}(t)\right)\right), \\
& \xi_{n}(t) \in S\left(t, x_{n}(t)\right), \\
\left\|\xi_{n}(t)-\xi_{n+1}(t)\right\| \leq & \left(1+\frac{1}{n+1}\right) H\left(S\left(t, x_{n}(t)\right), S\left(t, x_{n+1}(t)\right)\right) .
\end{aligned}
$$

Similarly, we have the following algorithms.

Algorithm 3.3. Suppose that $D, \lambda, g, f, N, T, S, F, A$ are the same as in Algorithm 3.2 and $J(t, z(t))=m(t, z(t))+K$ for all $t \in \Omega$ and measurable operator $z: \Omega \rightarrow D$, where $m: \Omega \times D \rightarrow E$ is a single-valued mapping and $K$ is a closed convex subset of $E$. For an arbitrarily chosen measurable mapping $x_{0}: \Omega \rightarrow D$, the sequences $\left\{x_{n}(t)\right\},\left\{u_{n}(t)\right\},\left\{v_{n}(t)\right\}$, and $\left\{\xi_{n}(t)\right\}$ are generated by a random iterative procedure

$$
\begin{aligned}
g\left(t, x_{n+1}(t)\right)=g\left(t, x_{n}(t)\right)-\lambda(t)\left\{g\left(t, x_{n}(t)\right)-m\left(t, v_{n}(t)\right)-P_{K}\left[g\left(t, x_{n}(t)\right)\right.\right. & \left.\left.-\rho(t)\left(N\left(t, f\left(t, x_{n}(t)\right), \xi_{n}(t)\right)+A\left(t, u_{n}(t)\right)\right)+m\left(t, v_{n}(t)\right)\right]\right\}, \\
& u_{n}(t) \in T\left(t, x_{n}(t)\right), \\
\left\|u_{n}(t)-u_{n+1}(t)\right\| \leq & \left(1+\frac{1}{n+1}\right) H\left(T\left(t, x_{n}(t)\right), T\left(t, x_{n+1}(t)\right)\right), \\
& v_{n}(t) \in F\left(t, x_{n}(t)\right), \\
\left\|v_{n}(t)-v_{n+1}(t)\right\| \leq & \left(1+\frac{1}{n+1}\right) H\left(F\left(t, x_{n}(t)\right), F\left(t, x_{n+1}(t)\right)\right), \\
& \xi_{n}(t) \in S\left(t, x_{n}(t)\right), \\
\left\|\xi_{n}(t)-\xi_{n+1}(t)\right\| \leq & \left(1+\frac{1}{n+1}\right) H\left(S\left(t, x_{n}(t)\right), S\left(t, x_{n+1}(t)\right)\right) .
\end{aligned}
$$


Algorithm 3.4. Let $D, \lambda, g, f, N, J, A$ be the same as in Algorithm 3.2 and let $T, S: \Omega \times$ $D \rightarrow E$ be two measurable single-valued mappings. For an arbitrarily chosen measurable mapping $x_{0}: \Omega \rightarrow D$, we can define the sequence $\left\{x_{n}(t)\right\}$ of measurable mappings by

$$
\begin{aligned}
g\left(t, x_{n+1}(t)\right) & \\
=g\left(t, x_{n}(t)\right)-\lambda(t)\left\{g\left(t, x_{n}(t)\right)-P_{J\left(t, x_{n}(t)\right)}[\right. & g\left(t, x_{n}(t)\right)-\rho(t)\left(N\left(t, f\left(t, x_{n}(t)\right), S\left(t, x_{n}(t)\right)\right)\right. \\
& \left.\left.\left.+A\left(t, T\left(t, x_{n}(t)\right)\right)\right)\right]\right\} .
\end{aligned}
$$

It is easy to see that if we suppose that the mapping $g: \Omega \times D \rightarrow E$ is expansive, then the inverse mapping $g^{-1}$ of $g$ exists and each $x_{n}(t)$ is computable for all $t \in \Omega$.

Now, we prove the existence of solutions of the problem (1.1) and the convergence of Algorithm 3.2.

THeORem 3.5. Let $D$ be a nonempty subset of a separable real Hilbert space $E$ and let $g: \Omega \times D \rightarrow E$ be a measurable mapping such that $g(\Omega \times D)$ is a closed set in E. Suppose that $N: \Omega \times E \times E \rightarrow E$ is a random $\kappa(t)$-Lipschitz continuous single mapping with respect to the third argument and $J: \Omega \times D \rightarrow P(E)$ is a random multivalued mapping such that $J(\Omega \times D) \subset g(\Omega \times D)$, for each $t \in \Omega$ and $x \in E, J(t, x)$ is nonempty closed convex and $P_{J(t, x)}$ is an $\eta(t)$-Lipschitz continuous random operator on $\Omega \times D$. Let $f: \Omega \times E \rightarrow E$ be $\delta(t)$-strongly monotone and $\sigma(t)$-Lipschitz continuous on $\Omega \times D$ with respect to the second argument of $N$ and $g$, and let $a: \Omega \times E \times E \rightarrow \mathbb{R}$ be a random $\beta(t)$-bounded bilinear function. Let random multivalued mappings $T, S: \Omega \times D \rightarrow C B(E), F: \Omega \times D \rightarrow C B(D)$ be $H$-Lipschitz continuous with respect to $g$ with measurable functions $\varsigma(t), \tau(t)$, and $\zeta(t)$, respectively. If for any $t \in \Omega$,

$$
\begin{gathered}
\nu(t)=\kappa(t) \tau(t)+\beta(t) \zeta(t)<\sigma(t), \\
0<\rho(t)<\frac{1-\eta(t) \zeta(t)}{\nu(t)}, \\
\delta(t)>v(t)(1-\eta(t) \zeta(t))+\sqrt{\eta(t) \zeta(t)(2-\eta(t) \zeta(t))\left(\sigma(t)^{2}-\nu(t)^{2}\right)}, \\
\left|\rho(t)-\frac{\delta(t)-\nu(t)(1-\eta(t) \zeta(t))}{\sigma(t)^{2}-\nu(t)^{2}}\right| \\
<\frac{\sqrt{(\delta(t)-\nu(t)(1-\eta(t) \zeta(t)))^{2}-\eta(t) \zeta(t)(2-\eta(t) \zeta(t))\left(\sigma(t)^{2}-\nu(t)^{2}\right)}}{\sigma(t)^{2}-\nu(t)^{2}},
\end{gathered}
$$

then for any $t \in \Omega$, there exist $x^{*}(t) \in D, u^{*}(t) \in T\left(t, x^{*}(t)\right), v^{*}(t) \in F\left(t, x^{*}(t)\right)$, and $\xi^{*}(t) \in S\left(t, x^{*}(t)\right)$ such that $\left(x^{*}(t), u^{*}(t), v^{*}(t), \xi^{*}(t)\right)$ is a solution of the problem (1.1) and

$g\left(t, x_{n}(t)\right) \longrightarrow g\left(t, x^{*}(t)\right), \quad u_{n}(t) \longrightarrow u^{*}(t), \quad v_{n}(t) \longrightarrow v^{*}(t), \quad \xi_{n}(t) \longrightarrow \xi^{*}(t) \quad$ as $n \longrightarrow \infty$,

where $\left\{x_{n}(t)\right\},\left\{u_{n}(t)\right\},\left\{v_{n}(t)\right\}$, and $\left\{\xi_{n}(t)\right\}$ are iterative sequences generated by Algorithm 3.2. 
Proof. It follows from (3.3), Lemma 2.11, and Definition 2.12 that

$$
\begin{aligned}
& \left\|g\left(t, x_{n+1}(t)\right)-g\left(t, x_{n}(t)\right)\right\| \leq(1-\lambda(t))\left\|g\left(t, x_{n}(t)\right)-g\left(t, x_{n-1}(t)\right)\right\| \\
& +\lambda(t) \| P_{J\left(t, v_{n}(t)\right)}\left[g\left(t, x_{n}(t)\right)-\rho(t)\left(N\left(t, f\left(t, x_{n}(t)\right), \xi_{n}(t)\right)+A\left(t, u_{n}(t)\right)\right)\right] \\
& \quad-P_{J\left(t, v_{n}(t)\right)}\left[g\left(t, x_{n-1}(t)\right)-\rho(t)\left(N\left(t, f\left(t, x_{n-1}(t)\right), \xi_{n-1}(t)\right)+A\left(t, u_{n-1}(t)\right)\right)\right] \| \\
& +\lambda(t) \| P_{J\left(t, v_{n}(t)\right)}\left[g\left(t, x_{n-1}(t)\right)-\rho(t)\left(N\left(t, f\left(t, x_{n-1}(t)\right), \xi_{n-1}(t)\right)+A\left(t, u_{n-1}(t)\right)\right)\right] \\
& \quad-P_{J\left(t, v_{n-1}(t)\right)}\left[g\left(t, x_{n-1}(t)\right)-\rho(t)\left(N\left(t, f\left(t, x_{n-1}(t)\right), \xi_{n-1}(t)\right)+A\left(t, u_{n-1}(t)\right)\right)\right] \| \\
& \leq \quad(1-\lambda(t))\left\|g\left(t, x_{n}(t)\right)-g\left(t, x_{n-1}(t)\right)\right\|+\lambda(t) \eta(t)\left\|v_{n}(t)-v_{n-1}(t)\right\| \\
& +\lambda(t)\left\|g\left(t, x_{n}(t)\right)-g\left(t, x_{n-1}(t)\right)-\rho(t)\left(N\left(t, f\left(t, x_{n}(t)\right), \xi_{n}(t)\right)-N\left(t, f\left(t, x_{n-1}(t)\right), \xi_{n}(t)\right)\right)\right\| \\
& +\rho(t) \lambda(t)\left\|N\left(t, f\left(t, x_{n-1}(t)\right), \xi_{n}(t)\right)-N\left(t, f\left(t, x_{n-1}(t)\right), \xi_{n-1}(t)\right)\right\| \\
& +\rho(t) \lambda(t)\left\|A\left(t, u_{n}(t)\right)-A\left(t, u_{n-1}(t)\right)\right\| .
\end{aligned}
$$

Since $f: \Omega \times E \rightarrow E$ is $\delta(t)$-strongly monotone and $\sigma(t)$-Lipschitz continuous with respect to the second argument of $N$ and $g, T, S$, and $F$ are $H$-Lipschitz continuous with respect to $g$ with measurable functions $\varsigma(t), \tau(t)$, and $\zeta(t)$, respectively, $N$ is $\kappa(t)$-Lipschitz continuous with respect to the third argument, and $a$ is a random $\beta(t)$-bounded bilinear function, we get

$$
\begin{gathered}
\left\|g\left(t, x_{n}(t)\right)-g\left(t, x_{n-1}(t)\right)-\rho(t)\left(N\left(t, f\left(t, x_{n}(t)\right), \xi_{n}(t)\right)-N\left(t, f\left(t, x_{n-1}(t)\right), \xi_{n}(t)\right)\right)\right\| \\
\leq \sqrt{1-2 \rho(t) \delta(t)+\rho(t)^{2} \sigma(t)^{2}}\left\|g\left(t, x_{n}(t)\right)-g\left(t, x_{n-1}(t)\right)\right\| \\
\left\|v_{n}(t)-v_{n-1}(t)\right\| \leq(1+\varepsilon) H\left(F\left(t, x_{n}(t)\right), F\left(t, x_{n-1}(t)\right)\right) \\
\leq(1+\varepsilon) \zeta(t)\left\|g\left(t, x_{n}(t)\right)-g\left(t, x_{n-1}(t)\right)\right\| \\
\begin{array}{c}
\left\|N\left(t, f\left(t, x_{n-1}(t)\right), \xi_{n}(t)\right)-N\left(t, f\left(t, x_{n-1}(t)\right), \xi_{n-1}(t)\right)\right\| \\
\leq \kappa(t)\left\|\xi_{n}(t)-\xi_{n-1}(t)\right\| \leq \kappa(t)(1+\varepsilon) H\left(S\left(t, x_{n}(t)\right), S\left(t, x_{n-1}(t)\right)\right) \\
\leq(1+\varepsilon) \kappa(t) \tau(t)\left\|g\left(t, x_{n}(t)\right)-g\left(t, x_{n-1}(t)\right)\right\|, \\
\left\|A\left(t, u_{n}(t)\right)-A\left(t, u_{n-1}(t)\right)\right\| \leq \beta(t)\left\|u_{n}(t)-u_{n-1}(t)\right\| \\
\leq(1+\varepsilon) \beta(t) H\left(T\left(t, x_{n}(t)\right), T\left(t, x_{n-1}(t)\right)\right) \\
\leq(1+\varepsilon) \beta(t) \varsigma(t)\left\|g\left(t, x_{n}(t)\right)-g\left(t, x_{n-1}(t)\right)\right\| .
\end{array}
\end{gathered}
$$

Using (3.9)-(3.12) in (3.8), for all $t \in \Omega$, we have

$$
\begin{aligned}
& \left\|g\left(t, x_{n+1}(t)\right)-g\left(t, x_{n}(t)\right)\right\| \\
& \leq\left\{1-\lambda(t)+\lambda(t)\left[\eta(t) \zeta(t)(1+\varepsilon)+\sqrt{1-2 \rho(t) \delta(t)+\rho(t)^{2} \sigma(t)^{2}}\right.\right. \\
& \quad+\rho(t)(1+\varepsilon)(\kappa(t) \tau(t)+\beta(t) \varsigma(t))]\} \\
& \quad \times\left\|g\left(t, x_{n}(t)\right)-g\left(t, x_{n-1}(t)\right)\right\|=\vartheta(t, \varepsilon)\left\|g\left(t, x_{n}(t)\right)-g\left(t, x_{n-1}(t)\right)\right\|,
\end{aligned}
$$


where

$$
\begin{aligned}
\vartheta(t, \varepsilon)= & 1-\lambda(t)(1-k(t, \varepsilon)), \\
k(t, \varepsilon)= & \eta(t) \zeta(t)(1+\varepsilon)+\sqrt{1-2 \rho(t) \delta(t)+\rho(t)^{2} \sigma(t)^{2}} \\
& +\rho(t)(1+\varepsilon)(\kappa(t) \tau(t)+\beta(t) \varsigma(t)) .
\end{aligned}
$$

Let $k(t)=\eta(t) \zeta(t)+\sqrt{1-2 \rho(t) \delta(t)+\rho(t)^{2} \sigma(t)^{2}}+\rho(t)(\kappa(t) \tau(t)+\beta(t) \varsigma(t)), \quad \vartheta(t)=1-$ $\lambda(t)(1-k(t))$. Then $k(t, \varepsilon) \rightarrow k(t), \vartheta(t, \varepsilon) \rightarrow \vartheta(t)$ as $\varepsilon \rightarrow 0$. From condition (3.6), we know that $0<\vartheta(t)<1$ for all $t \in \Omega$ and so $0<\vartheta(t, \varepsilon)<1$ for $\varepsilon$ sufficiently small and all $t \in \Omega$. It follows from (3.13) that $\left\{g\left(t, x_{n}(t)\right)\right\}$ is a Cauchy sequence in $g(\Omega \times D)$. Since $g(\Omega \times D)$ is closed in $E$, there exists a measurable mapping $x^{*}: \Omega \rightarrow D$ such that for $t \in \Omega$,

$$
g\left(t, x_{n}(t)\right) \longrightarrow g\left(t, x^{*}(t)\right) \quad \text { as } n \longrightarrow \infty
$$

By (3.10)-(3.12), we know that $\left\{u_{n}(t)\right\},\left\{v_{n}(t)\right\}$, and $\left\{\xi_{n}(t)\right\}$ are also Cauchy sequences in $E$. Let

$$
u_{n}(t) \longrightarrow u^{*}(t), \quad v_{n}(t) \longrightarrow v^{*}(t), \quad \xi_{n}(t) \longrightarrow \xi^{*}(t) \quad \text { as } n \longrightarrow \infty
$$

Now we show that $u^{*}(t) \in T\left(t, x^{*}(t)\right)$. In fact, we have

$$
\begin{aligned}
d\left(u^{*}(t), T\left(t, x^{*}(t)\right)\right) & =\inf \left\{\left\|u^{*}(t)-y\right\|: y \in T\left(t, x^{*}(t)\right)\right\} \\
& \leq\left\|u^{*}(t)-u_{n}(t)\right\|+d\left(u_{n}(t), T\left(t, x^{*}(t)\right)\right) \\
& \leq\left\|u^{*}(t)-u_{n}(t)\right\|+H\left(T\left(t, x_{n}(t)\right), T\left(t, x^{*}(t)\right)\right) \\
& \leq\left\|u^{*}(t)-u_{n}(t)\right\|+\varsigma(t)\left\|g\left(t, x_{n}(t)\right)-g\left(t, x^{*}(t)\right)\right\| \longrightarrow 0 .
\end{aligned}
$$

This implies that $u^{*}(t) \in T\left(t, x^{*}(t)\right)$. Similarly, we have $v^{*}(t) \in F\left(t, x^{*}(t)\right)$ and $\xi^{*}(t) \in$ $S\left(t, x^{*}(t)\right)$.

Next, we prove that

$$
g\left(t, x^{*}(t)\right)=P_{J\left(t, v^{*}(t)\right)}\left[g\left(t, x^{*}(t)\right)-\rho(t)\left(N\left(t, f\left(t, x^{*}(t)\right), \xi^{*}(t)\right)+A\left(t, u^{*}(t)\right)\right)\right] .
$$

Indeed, from (3.3), we know that it is enough to prove that

$$
\begin{aligned}
\lim _{n \rightarrow \infty} P_{J\left(t, v_{n}(t)\right)}\left[g\left(t, x_{n}(t)\right)-\rho(t)\left(N\left(t, f\left(t, x_{n}(t)\right), \xi_{n}(t)\right)+A\left(t, u_{n}(t)\right)\right)\right] \\
\quad=P_{J\left(t, v^{*}(t)\right)}\left[g\left(t, x^{*}(t)\right)-\rho(t)\left(N\left(t, f\left(t, x^{*}(t)\right), \xi^{*}(t)\right)+A\left(t, u^{*}(t)\right)\right)\right] .
\end{aligned}
$$


It follows from Lemma 2.11 and Definition 2.12 that

$$
\begin{aligned}
& \| P_{J\left(t, v_{n}(t)\right)}\left[g\left(t, x_{n}(t)\right)-\rho(t)\left(N\left(t, f\left(t, x_{n}(t)\right), \xi_{n}(t)\right)+A\left(t, u_{n}(t)\right)\right)\right] \\
& -P_{J\left(t, v^{*}(t)\right)}\left[g\left(t, x^{*}(t)\right)-\rho(t)\left(N\left(t, f\left(t, x^{*}(t)\right), \xi^{*}(t)\right)+A\left(t, u^{*}(t)\right)\right)\right] \| \\
& \leq \| P_{J\left(t, v_{n}(t)\right)}\left[g\left(t, x_{n}(t)\right)-\rho(t)\left(N\left(t, f\left(t, x_{n}(t)\right), \xi_{n}(t)\right)+A\left(t, u_{n}(t)\right)\right)\right] \\
& -P_{J\left(t, v_{n}(t)\right)}\left[g\left(t, x^{*}(t)\right)-\rho(t)\left(N\left(t, f\left(t, x^{*}(t)\right), \xi^{*}(t)\right)+A\left(t, u^{*}(t)\right)\right)\right] \| \\
& +\| P_{J\left(t, v_{n}(t)\right)}\left[g\left(t, x^{*}(t)\right)-\rho(t)\left(N\left(t, f\left(t, x^{*}(t)\right), \xi^{*}(t)\right)+A\left(t, u^{*}(t)\right)\right)\right] \\
& -P_{J\left(t, v^{*}(t)\right)}\left[g\left(t, x^{*}(t)\right)-\rho(t)\left(N\left(t, f\left(t, x^{*}(t)\right), \xi^{*}(t)\right)+A\left(t, u^{*}(t)\right)\right)\right] \| \\
& \leq\left\|g\left(t, x_{n}(t)\right)-g\left(t, x^{*}(t)\right)-\rho(t)\left(N\left(t, f\left(t, x_{n}(t)\right), \xi_{n}(t)\right)-N\left(t, f\left(t, x^{*}(t)\right), \xi_{n}(t)\right)\right)\right\| \\
& +\rho(t)\left\|N\left(t, f\left(t, x^{*}(t)\right), \xi_{n}(t)\right)-N\left(t, f\left(t, x^{*}(t)\right), \xi^{*}(t)\right)\right\| \\
& +\rho(t)\left\|A\left(t, u_{n}(t)\right)-A\left(t, u^{*}(t)\right)\right\|+\eta(t)\left\|v_{n}(t)-v^{*}(t)\right\| \\
& \leq \sqrt{1-2 \rho(t) \delta(t)+\rho(t)^{2} \sigma(t)^{2}}\left\|g\left(t, x_{n}(t)\right)-g\left(t, x^{*}(t)\right)\right\| \\
& +\rho(t) \kappa(t)\left\|\xi_{n}(t)-\xi^{*}(t)\right\|+\rho(t) \beta(t)\left\|u_{n}(t)-u^{*}(t)\right\| \\
& +\eta(t)\left\|v_{n}(t)-v^{*}(t)\right\| \text {. }
\end{aligned}
$$

This implies that (3.19) holds and so (3.18) is true. This completes the proof.

From Theorem 3.5, we can obtain the following results.

Theorem 3.6. Let D, $g, N, f, T, F, S$, and a be the same as in Theorem 3.5. Suppose that $K$ is a nonempty closed convex subset of $E$ and that $m: \Omega \times D \rightarrow E$ is an $\eta(t) / 2$-Lipschitz continuous random operator. If there exists a measurable mapping $\rho: \Omega \rightarrow(0,+\infty)$ such that condition (3.6) holds, then for any $t \in \Omega$ there exist $x^{*}(t) \in D, u^{*}(t) \in T\left(t, x^{*}(t)\right), v^{*}(t) \in$ $F\left(t, x^{*}(t)\right)$, and $\xi^{*}(t) \in S\left(t, x^{*}(t)\right)$ such that $\left(x^{*}(t), u^{*}(t), v^{*}(t), \xi^{*}(t)\right)$ is a solution of the problem (1.3) and

$$
g\left(t, x_{n}(t)\right) \longrightarrow g\left(t, x^{*}(t)\right), \quad u_{n}(t) \longrightarrow u^{*}(t), \quad v_{n}(t) \longrightarrow v^{*}(t), \quad \xi_{n}(t) \longrightarrow \xi^{*}(t)
$$

as $n \rightarrow \infty$, where $\left\{x_{n}(t)\right\},\left\{u_{n}(t)\right\},\left\{v_{n}(t)\right\}$, and $\left\{\xi_{n}(t)\right\}$ are iterative sequences generated by Algorithm 3.3.

Proof. The conclusion follows from Lemma 2.13 and Theorem 3.5.

Remark 3.7. If $J(t, x) \equiv K$ for all $t \in \Omega$ and $x \in D$, then from Theorem 3.5, we can obtain the corresponding result.

Theorem 3.8. Let $D, g, N, f$, and $a$ be the same as in Theorem 3.5. Suppose that $J: \Omega \times$ $D \rightarrow P(E)$ is a random multivalued mapping such that $J(\Omega \times D) \subset g(\Omega \times D)$, for each $t \in$ $\Omega$ and $x \in E, J(t, x)$ is nonempty closed convex and $P_{J(t, x)}$ is an $\eta(t)$-Lipschitz continuous random operator on $\Omega \times D$ with respect to $g$, and $T, S: \Omega \times D \rightarrow E$ are Lipschitz continuous 
on $\Omega \times D$ with respect to $g$ with measurable functions $\varsigma(t)$ and $\tau(t)$, respectively. If there exists a measurable mapping $\rho: \Omega \rightarrow(0,+\infty)$ such that

$$
\begin{gathered}
v(t)=\kappa(t) \tau(t)+\beta(t) \varsigma(t)<\sigma(t), \\
\rho(t)<\frac{1-\eta(t)}{\nu(t)}, \\
\delta(t)>\nu(t)(1-\eta(t))+\sqrt{\eta(t)(2-\eta(t))\left(\sigma(t)^{2}-\nu(t)^{2}\right)} \\
\left|\rho(t)-\frac{\delta(t)-\nu(t)(1-\eta(t))}{\sigma(t)^{2}-\nu(t)^{2}}\right|<\frac{\sqrt{(\delta(t)-\nu(t)(1-\eta(t)))^{2}-\eta(t)(2-\eta(t))\left(\sigma(t)^{2}-\nu(t)^{2}\right)}}{\sigma(t)^{2}-\nu(t)^{2}},
\end{gathered}
$$

then there exists a unique measurable mapping $x^{*}: \Omega \rightarrow D$, which is the solution of the problem (1.5) and $\lim _{n \rightarrow \infty} g\left(t, x_{n}(t)\right)=g\left(t, x^{*}(t)\right)$ for all $t \in \Omega$, where $\left\{x_{n}(t)\right\}$ is the iterative sequence generated by Algorithm 3.4 .

Proof. From Theorem 3.5, we know that there exists a measurable mapping $x^{*}: \Omega \rightarrow D$ such that it is the solution of the problem (1.5) and $\lim _{n \rightarrow \infty} g\left(t, x_{n}(t)\right)=g\left(t, x^{*}(t)\right)$ for all $t \in \Omega$. Now we prove that $x^{*}: \Omega \rightarrow D$ is a unique solution of the problem (1.5). In fact, if $x: \Omega \rightarrow D$ is also a solution of the problem (1.5), then

$$
g(t, x(t))=P_{J(t, x(t))}[g(t, x(t))-\rho(t)(N(t, f(t, x(t)), S(t, x(t)))+A(t, T(t, x(t))))] .
$$

By the proof of (3.13) in Theorem 3.5, we have

$$
\begin{aligned}
& \left\|g(t, x(t))-g\left(t, x^{*}(t)\right)\right\| \\
& \leq\left\|g(t, x(t))-g\left(t, x^{*}(t)\right)-\rho(t)\left(N(t, f(t, x(t)), S(t, x(t)))-N\left(t, f\left(t, x^{*}(t)\right), S(t, x(t))\right)\right)\right\| \\
& \quad+\rho(t)\left\|N\left(t, f\left(t, x^{*}(t)\right), S(t, x(t))\right)-N\left(t, f\left(t, x^{*}(t)\right), S\left(t, x^{*}(t)\right)\right)\right\| \\
& \quad+\rho(t)\left\|A(t, T(t, x(t)))-A\left(t, T\left(t, x^{*}(t)\right)\right)\right\|+\eta(t)\left\|g(t, x(t))-g\left(t, x^{*}(t)\right)\right\| \\
& \leq \omega(t)\left\|g(t, x(t))-g\left(t, x^{*}(t)\right)\right\|,
\end{aligned}
$$

where $\omega(t)=\eta(t)+\sqrt{1-2 \rho(t) \delta(t)+\rho(t)^{2} \sigma(t)^{2}}+\rho(t)(\kappa(t) \tau(t)+\beta(t) \varsigma(t))$. It follows from (3.22) that $0<\omega(t)<1$ and so $x^{*}(t)=x(t)$ for all $t \in \Omega$. This completes the proof.

Remark 3.9. From Theorems 3.5-3.8, we get several known results of $[4,5,10,13,15,17$, 20] as special cases.

\section{Random perturbed algorithm and stability}

In this section, we construct a new perturbed iterative algorithm for solving the random generalized nonlinear implicit quasi-variational inequality problem (1.5) and prove the 
convergence and stability of the iterative sequence generated by the perturbed iterative algorithm.

For our purpose, we first review the following concept and lemma.

Definition 4.1. Let $S$ be a self-map of $E, x_{0} \in E$, and let $x_{n+1}=h\left(S, x_{n}\right)$ define an iteration procedure which yields a sequence of points $\left\{x_{n}\right\}_{n=0}^{\infty}$ in $E$. Suppose that $\{x \in E: S x=$ $x\} \neq \varnothing$ and that $\left\{x_{n}\right\}_{n=0}^{\infty}$ converges to a fixed point $x^{*}$ of $S$. Let $\left\{u_{n}\right\} \subset E$ and let $\epsilon_{n}=$ $\left\|u_{n+1}-h\left(S, u_{n}\right)\right\|$. If $\lim \epsilon_{n}=0$ implies that $u_{n} \rightarrow x^{*}$, then the iteration procedure defined by $x_{n+1}=h\left(S, x_{n}\right)$ is said to be $S$-stable or stable with respect to $S$.

Lemma 4.2 [23]. Let $\left\{\gamma_{n}\right\}$ be a nonnegative real sequence and let $\left\{\lambda_{n}\right\}$ be a real sequence in $[0,1]$ such that $\sum_{n=0}^{\infty} \lambda_{n}=\infty$. If there exists a positive integer $n_{1}$ such that

$$
\gamma_{n+1} \leq\left(1-\lambda_{n}\right) \gamma_{n}+\lambda_{n} \sigma_{n}, \quad \forall n \geq n_{1},
$$

where $\sigma_{n} \geq 0$ for all $n \geq 0$ and $\sigma_{n} \rightarrow 0$ as $n \rightarrow \infty$, then $\lim _{n \rightarrow \infty} \gamma_{n}=0$.

Algorithm 4.3. Let $D$ be a nonempty subset of a separable real Hilbert space $E$, let $g$ : $\Omega \times D \rightarrow E, f: \Omega \times E \rightarrow E, N: \Omega \times E \times E \rightarrow E$, and $T, S: \Omega \times D \rightarrow E$ be measurable singlevalued mappings, and let $J: \Omega \times D \rightarrow P(E)$ be a random multivalued mapping such that for each $t \in \Omega$ and $x \in D, J(t, x)$ is closed convex and $J(\Omega \times D) \subset g(\Omega \times D)$. For a given measurable mapping $x_{0}: \Omega \rightarrow D$, the perturbed iterative sequence $\left\{x_{n}(t)\right\}$ is defined by

$$
\begin{aligned}
g\left(t, x_{n+1}(t)\right)= & \left(1-\alpha_{n}(t)\right) g\left(t, x_{n}(t)\right) \\
+ & +\alpha_{n}(t) P_{J\left(t, y_{n}(t)\right)}\left[g\left(t, y_{n}(t)\right)-\rho(t)\left(N\left(t, f\left(t, y_{n}(t)\right), S\left(t, y_{n}(t)\right)\right)\right.\right. \\
& \left.\left.+A\left(t, T\left(t, y_{n}(t)\right)\right)\right)\right], \\
g\left(t, y_{n}(t)\right)= & \left(1-\beta_{n}(t)\right) g\left(t, x_{n}(t)\right) \\
+ & +\beta_{n}(t) P_{J\left(t, x_{n}(t)\right)}\left[g\left(t, x_{n}(t)\right)-\rho(t)\left(N\left(t, f\left(t, x_{n}(t)\right), S\left(t, x_{n}(t)\right)\right)\right.\right. \\
& \left.\left.+A\left(t, T\left(t, x_{n}(t)\right)\right)\right)\right],
\end{aligned}
$$

for $n=0,1,2, \ldots$, where $\rho$ and $A$ are the same as in Lemma 3.1, and $\left\{\alpha_{n}(t)\right\}$ and $\left\{\beta_{n}(t)\right\}$ satisfy the following conditions:

$$
0 \leq \alpha_{n}(t), \quad \beta_{n}(t) \leq 1 \quad(n \geq 0), \quad \sum_{n=0}^{\infty} \alpha_{n}(t)=\infty, \quad \forall t \in \Omega .
$$

Let $\left\{u_{n}(t)\right\}$ be any sequence in $E$ and define $\left\{\epsilon_{n}(t)\right\}$ by

$$
\begin{gathered}
\epsilon_{n}(t)=\| g\left(t, u_{n+1}(t)\right) \\
-\left\{\left(1-\alpha_{n}(t)\right) g\left(t, u_{n}(t)\right)+\alpha_{n}(t) P_{J\left(t, v_{n}(t)\right)}\left[g\left(t, v_{n}(t)\right)\right.\right. \\
\left.\left.\quad-\rho(t)\left(N\left(t, f\left(t, v_{n}(t)\right), S\left(t, v_{n}(t)\right)\right)+A\left(t, T\left(t, v_{n}(t)\right)\right)\right)\right]\right\} \|, \\
g\left(t, v_{n}(t)\right)=\left(1-\beta_{n}(t)\right) g\left(t, u_{n}(t)\right) \\
+\beta_{n} P_{J\left(t, u_{n}(t)\right)}\left[g\left(t, u_{n}(t)\right)-\rho(t)\left(N\left(t, f\left(t, u_{n}(t)\right), S\left(t, u_{n}(t)\right)\right)\right.\right. \\
\left.\left.+A\left(t, T\left(t, u_{n}(t)\right)\right)\right)\right]
\end{gathered}
$$

for $n=0,1,2, \ldots$ 
Theorem 4.4. Let $D$ be a nonempty subset of a separable real Hilbert space $E$ and let $g$ : $\Omega \times D \rightarrow E$ be a measurable mapping such that $g(\Omega \times D)$ is a closed set in E. Suppose that $N: \Omega \times E \times E \rightarrow E$ is a random $\kappa(t)$-Lipschitz continuous single mapping with respect to the third argument, and $f: \Omega \times E \rightarrow E$ is $\delta(t)$-strongly monotone and $\sigma(t)$-Lipschitz continuous on $\Omega \times D$ with respect to the second argument of $N$ and $g$. Let $J: \Omega \times D \rightarrow P(E)$ be a random multivalued mapping such that $J(\Omega \times D) \subset g(\Omega \times D)$ for each $t \in \Omega$ and $x \in E, J(t, x)$ is nonempty closed convex, and $P_{J(t, x)}$ is an $\eta(t)$-Lipschitz continuous random operator on $\Omega \times D$ with respect to $g$, and let $T, S: \Omega \times D \rightarrow E$ be Lipschitz continuous with respect to $g$ with measurable functions $\varsigma(t)$ and $\tau(t)$, respectively. If $a: \Omega \times E \times E \rightarrow \mathbb{R}$ is a random $\beta(t)$-bounded bilinear function such that (3.22) holds, then

(i) if $\lim _{n \rightarrow \infty} \sum_{i=0}^{n} \ln \left[1-\alpha_{i}(1-q)\right]=-\infty$, where $q=1-\tau+(1+\lambda \rho \alpha)^{-1}(\delta+\rho \nu)$, then the sequence $\left\{x_{n}(t)\right\}$ generated by (4.2) converges strongly to the unique solution $x(t)$ of the problem (1.5) for all $t \in \Omega$;

(ii) moreover, if for any $t \in \Omega, 0<a \leq \alpha_{n}(t)$, then $\lim u_{n}(t)=x(t)$ if and only if $\lim \epsilon_{n}(t)=0$, where $\epsilon_{n}(t)$ is defined by (4.4).

Proof. From Theorem 3.8, we know that there exists a unique solution $x(t)$ of the problem (1.5) and so

$$
g(t, x(t))=P_{J(t, x(t))}[g(t, x(t))-\rho(t)(N(t, f(t, x(t)), S(t, x(t)))+A(t, T(t, x(t))))] .
$$

From (4.2), (4.5), Lemma 2.11, and Definition 2.12, it follows that

$$
\begin{aligned}
& \left\|g\left(t, x_{n+1}(t)\right)-g(t, x(t))\right\| \\
& \leq\left(1-\alpha_{n}(t)\right)\left\|g\left(t, x_{n}(t)\right)-g(t, x(t))\right\| \\
& +\alpha_{n}(t)\left\{\| P_{J\left(t, y_{n}(t)\right)}\left[g\left(t, y_{n}(t)\right)-\rho(t)\left(N\left(t, f\left(t, y_{n}(t)\right), S\left(t, y_{n}(t)\right)\right)\right.\right.\right. \\
& \left.\left.+A\left(t, T\left(t, y_{n}(t)\right)\right)\right)\right] \\
& -P_{J\left(t, y_{n}(t)\right)}[g(t, x(t))-\rho(t)(N(t, f(t, x(t)), S(t, x(t)))+A(t, T(t, x(t))))] \| \\
& +\| P_{J\left(t, y_{n}(t)\right)}[g(t, x(t))-\rho(t)(N(t, f(t, x(t)), S(t, x(t)))+A(t, T(t, x(t))))] \\
& \left.-P_{J(t, x(t))}[g(t, x(t))-\rho(t)(N(t, f(t, x(t)), S(t, x(t)))+A(t, T(t, x(t))))] \mid\right\} \\
& \leq\left(1-\alpha_{n}(t)\right)\left\|g\left(t, x_{n}(t)\right)-g(t, x(t))\right\| \\
& +\alpha_{n}(t)\left\{\| g\left(t, y_{n}(t)\right)-g(t, x(t))-\rho(t)\left(N\left(t, f\left(t, y_{n}(t)\right), S\left(t, y_{n}(t)\right)\right)\right.\right. \\
& \left.-N\left(t, f(t, x(t)), S\left(t, y_{n}(t)\right)\right)\right) \| \\
& +\rho(t)\left\|N\left(t, f(t, x(t)), S\left(t, y_{n}(t)\right)\right)-N(t, f(t, x(t)), S(t, x(t)))\right\| \\
& +\rho(t)\left\|A\left(t, T\left(t, y_{n}(t)\right)\right)-A(t, T(t, x(t)))\right\| \\
& \left.+\eta(t)\left\|g\left(t, y_{n}(t)\right)-g(t, x(t))\right\|\right\} \text {. }
\end{aligned}
$$


By (4.6) and the assumption of $N, f, a, S, T$, we obtain

$$
\begin{aligned}
\left\|g\left(t, x_{n+1}(t)\right)-g(t, x(t))\right\| \leq & \left(1-\alpha_{n}(t)\right)\left\|g\left(t, x_{n}(t)\right)-g(t, x(t))\right\| \\
& +\alpha_{n}(t) q(t)\left\|g\left(t, y_{n}(t)\right)-g(t, x(t))\right\|,
\end{aligned}
$$

where

$$
q(t)=\sqrt{1-2 \rho(t) \delta(t)+\rho(t)^{2} \sigma(t)^{2}}+\rho(t)(\kappa(t) \tau(t)+\beta(t) \varsigma(t))+\eta(t) .
$$

Similarly, we have

$$
\begin{aligned}
\left\|g\left(t, y_{n}(t)\right)-g(t, x(t))\right\| \leq & \left(1-\beta_{n}(t)\right)\left\|g\left(t, x_{n}(t)\right)-g(t, x(t))\right\| \\
& +\beta_{n}(t) q(t)\left\|g\left(t, x_{n}(t)\right)-g(t, x(t))\right\| .
\end{aligned}
$$

Combining (4.7) and (4.9), we obtain

$$
\left\|g\left(t, x_{n+1}(t)\right)-g(t, x(t))\right\| \leq\left[1-\alpha_{n}(t)(1-q(t))\left(1+\beta_{n}(t)\right)\right]\left\|g\left(t, x_{n}(t)\right)-g(t, x(t))\right\| .
$$

Condition (3.22) implies that $0<q(t)<1$, and so from (4.10), we have

$$
\begin{aligned}
& \left\|g\left(t, x_{n+1}(t)\right)-g(t, x(t))\right\| \\
& \quad \leq\left[1-\alpha_{n}(t)(1-q(t))\right]\left\|g\left(t, x_{n}(t)\right)-g(t, x(t))\right\| \\
& \quad \leq \cdots \leq \prod_{i=0}^{n}\left[1-\alpha_{i}(t)(1-q(t))\right]\left\|g\left(t, x_{0}(t)\right)-g(t, x(t))\right\| .
\end{aligned}
$$

Since

$$
\lim _{n \rightarrow \infty} \sum_{i=0}^{n} \ln \left[1-\alpha_{i}(t)(1-q(t))\right]=-\infty, \quad \text { i.e., } \lim _{n \rightarrow \infty} \prod_{i=0}^{n}\left[1-\alpha_{i}(t)(1-q(t))\right]=0,
$$

we know that $g\left(t, x_{n}(t)\right) \rightarrow g(t, x(t))$ as $n \rightarrow \infty$.

Now we prove conclusion (ii). By (4.4), we obtain

$$
\begin{aligned}
& \left\|g\left(t, u_{n+1}(t)\right)-g(t, x(t))\right\| \\
& \leq \|\left(1-\alpha_{n}(t)\right) g\left(t, u_{n}(t)\right) \\
& \quad+\alpha_{n}(t) P_{J\left(t, v_{n}(t)\right)}\left[g\left(t, v_{n}(t)\right)-\rho(t)\left(N\left(t, f\left(t, v_{n}(t)\right), S\left(t, v_{n}(t)\right)\right)+A\left(t, T\left(t, v_{n}(t)\right)\right)\right)\right] \\
& \quad-g(t, x(t)) \|+\epsilon_{n}(t) .
\end{aligned}
$$

As the proof of inequality (4.10), we have

$$
\begin{gathered}
\|\left(1-\alpha_{n}(t)\right) g\left(t, u_{n}(t)\right) \\
+\alpha_{n}(t) P_{J\left(t, v_{n}(t)\right)}\left[g\left(t, v_{n}(t)\right)-\rho(t)\left(N\left(t, f\left(t, v_{n}(t)\right), S\left(t, v_{n}(t)\right)\right)\right.\right. \\
\left.\left.+A\left(t, T\left(t, v_{n}(t)\right)\right)\right)\right]-g(t, x(t)) \| \\
\leq\left[1-\alpha_{n}(t)(1-q(t))\right]\left\|g\left(t, u_{n}(t)\right)-g(t, x(t))\right\| .
\end{gathered}
$$


Since $0<a \leq \alpha_{n}(t)$ for all $t \in \Omega$, by (4.13) and (4.14), we have

$$
\begin{aligned}
\left\|g\left(t, u_{n+1}(t)\right)-g(t, x(t))\right\| \leq & {\left[1-\alpha_{n}(t)(1-q(t))\right]\left\|g\left(t, u_{n}(t)\right)-g(t, x(t))\right\| } \\
& +\alpha_{n}(t)(1-q(t)) \cdot \frac{\epsilon_{n}(t)}{a(1-q(t))} .
\end{aligned}
$$

Suppose that for any $t \in \Omega, \lim \epsilon_{n}(t)=0$. Then from $\sum_{n=0}^{\infty} \alpha_{n}(t)=\infty$ and Lemma 4.2, we have $\lim g\left(t, u_{n}(t)\right)=g(t, x(t))$ and so $\lim u_{n}(t)=x(t)$.

Conversely, if $\lim u_{n}(t)=x(t)$ for all $t \in \Omega$, then we get

$$
\begin{aligned}
\epsilon_{n}(t) \leq & \left\|g\left(t, u_{n+1}(t)\right)-g(t, x(t))\right\| \\
+ & \|\left(1-\alpha_{n}(t)\right) g\left(t, u_{n}(t)\right) \\
& \quad+\alpha_{n}(t) P_{J\left(t, v_{n}(t)\right)}\left[g\left(t, v_{n}(t)\right)-\rho(t)\left(N\left(t, f\left(t, v_{n}(t)\right), S\left(t, v_{n}(t)\right)\right)\right.\right. \\
& \left.\left.+A\left(t, T\left(t, v_{n}(t)\right)\right)\right)\right]-g(t, x(t)) \| \\
\leq & \left\|g\left(t, u_{n+1}(t)\right)-g(t, x(t))\right\| \\
& +\left[1-\alpha_{n}(t)(1-q(t))\right]\left\|g\left(t, u_{n}(t)\right)-g(t, x(t))\right\| \longrightarrow 0,
\end{aligned}
$$

as $n \rightarrow \infty$. This completes the proof.

Remark 4.5. If $J(t, x)=m(t, x)+K$ for all $t \in \Omega$ and $x \in D$, where $m: \Omega \times D \rightarrow E$ is an $\eta(t) / 2$-Lipschitz continuous random mapping and $K$ is a closed convex subset of $E$, then we can obtain the same results as in Theorem 4.4 .

\section{Acknowledgments}

The author acknowledges the referees for their valuable suggestions and the support of the Educational Science Foundation of Sichuan, Sichuan, China (2004C018).

\section{References}

[1] R. P. Agarwal, N.-J. Huang, and Y. J. Cho, Generalized nonlinear mixed implicit quasi-variational inclusions with set-valued mappings, Journal of Inequalities and Applications 7 (2002), no. 6, $807-828$.

[2] A. T. Bharucha-Reid, Random Integral Equations, Academic Press, New York, 1972.

[3] S. S. Chang, Fixed Point. Theory and Applications, Chongqing, Chongqing, 1984.

[4] _ Variational Inequality and Complementarity Problem Theory with Applications, Shanghai Scientific and Technological Literature, Shanghai, 1991.

[5] S. S. Chang and N.-J. Huang, Generalized random multivalued quasi-complementarity problems, Indian Journal of Mathematics 35 (1993), no. 3, 305-320.

[6] __ Random generalized set-valued quasi-complementarity problems, Acta Mathematicae Applicatae Sinica 16 (1993), 396-405.

[7] S. S. Chang and Y. G. Zhu, Problems concerning a class of random variational inequalities and random quasivariational inequalities, Journal of Mathematical Research and Exposition 9 (1989), no. 3, 385-393 (Chinese).

[8] Y. J. Cho, N.-J. Huang, and S. M. Kang, Random generalized set-valued strongly nonlinear implicit quasi-variational inequalities, Journal of Inequalities and Applications 5 (2000), no. 5, 515-531. 
[9] Y. J. Cho, S. H. Shim, N.-J. Huang, and S. M. Kang, Generalized strongly nonlinear implicit quasivariational inequalities for fuzzy mappings, Set Valued Mappings with Applications in Nonlinear Analysis, Ser. Math. Anal. Appl., vol. 4, Taylor \& Francis, London, 2002, pp. 63-77.

[10] X. P. Ding, Generalized quasi-variational-like inclusions with nonconvex functionals, Applied Mathematics and Computation 122 (2001), no. 3, 267-282.

[11] A. Ganguly and K. Wadhwa, On random variational inequalities, Journal of Mathematical Analysis and Applications 206 (1997), no. 1, 315-321.

[12] C. J. Himmelberg, Measurable relations, Fundamenta Mathematicae 87 (1975), 53-72.

[13] N.-J. Huang, On the generalized implicit quasivariational inequalities, Journal of Mathematical Analysis and Applications 216 (1997), no. 1, 197-210.

[14] _ Random generalized nonlinear variational inclusions for random fuzzy mappings, Fuzzy Sets and Systems 105 (1999), no. 3, 437-444.

[15] N.-J. Huang and Y. J. Cho, Random completely generalized set-valued implicit quasi-variational inequalities, Positivity 3 (1999), no. 3, 201-213.

[16] N.-J. Huang, X. Long, and Y. J. Cho, Random completely generalized nonlinear variational inclusions with non-compact valued random mappings, Bulletin of the Korean Mathematical Society 34 (1997), no. 4, 603-615.

[17] H.-Y. Lan, N.-J. Huang, and Y. J. Cho, A new method for nonlinear variational inequalities with multi-valued mappings, Archives of Inequalities and Applications 2 (2004), no. 1, 73-84.

[18] H.-Y. Lan, J. K. Kim, and N.-J. Huang, On the generalized nonlinear quasi-variational inclusions involving non-monotone set-valued mappings, Nonlinear Functional Analysis and Applications 9 (2004), no. 3, 451-465.

[19] M. A. Noor and S. A. Elsanousi, Iterative algorithms for random variational inequalities, Panamerican Mathematical Journal 3 (1993), no. 1, 39-50.

[20] A. H. Siddiqi and Q. H. Ansari, Strongly nonlinear quasivariational inequalities, Journal of Mathematical Analysis and Applications 149 (1990), no. 2, 444-450.

[21] C. P. Toskos and W. J. Padgett, Random Integral Equation with Applications in Life Sciences and Engineering, Academic Press, New York, 1974.

[22] R. U. Verma, A class of projection-contraction methods applied to monotone variational inequalities, Applied Mathematics Letters 13 (2000), no. 8, 55-62.

[23] X. L. Weng, Fixed point iteration for local strictly pseudo-contractive mapping, Proceedings of the American Mathematical Society 113 (1991), no. 3, 727-731.

[24] X.-Z. Yuan, X. Luo, and G. Li, Random approximations and fixed point theorems, Journal of Approximation Theory 84 (1996), no. 2, 172-187.

[25] H. Y. Zhou, Y. J. Cho, and S. M. Kang, Iterative approximations for solutions of nonlinear equations involving non-self-mappings, Journal of Inequalities and Applications 6 (2001), no. 6, 577-597.

Heng-You Lan: Department of Mathematics, Sichuan University of Science and Engineering,

Zigong, Sichuan 643000, China

E-mail addresses: hengyoulan@163.com; lanhengyou@suse.edu.cn 